\title{
Research Progress on the Occurrence Mechanism of Renal Interstitial Fibrosis
}

\author{
Qi Jiang, Yingying Liu*
}

\author{
Department of Nephrology, China-Japan Union Hospital of Jilin University, Changchun, 130033, China \\ ${ }^{*}$ Corresponding author
}

Keywords: Research Progress, Occurrence Mechanism, Renal Interstitial

\begin{abstract}
Renal fibrosis refers to the increase of interstitial cells and interstitial cells under various pathogenic factors such as inflammation, injury and drugs, especially the increase of matrix protein synthesis, and the suppression of matrix degradation resulting in extracellular matrix ECM) caused a large accumulation of glomerular sclerosis and tubulointerstitial fibrosis. It is a common pathological manifestation of renal disease progression to end-stage renal failure. The current study confirms that the correlation between renal interstitial fibrosis and renal dysfunction, which is more closely related to glomerulosclerosis and renal dysfunction. To further study the molecular mechanism of renal interstitial fibrosis and explore effective prevention and treatment measures is of great significance for delaying the progression of endstage renal disease (ESRD) and prolonging the life of patients.
\end{abstract}

\section{Introduction}

Ten years ago, Strutz et al. first reported the occurrence of EMT in renal fibrosis. In the rat model of anti-tubulointerstitial disease, the authors used FSP1 (fibroblast-specific protein-1) as a marker and found that tubular epithelial cells express FSP1, which is usually found only in fibroblasts Cells are expressed but epithelial cells are not expressed. So they put forward the hypothesis of EMT. Subsequently, Lan et. al. Also demonstrated on the morphological and cellular phenotypic characteristics the presence of EMT in residual kidneys after 5/6 nephrectomy. Consistent with animal experiments, EMT is also present in human renal biopsies. Rastaldi et al. [1] reported the renal biopsy results of 133 patients with different renal diseases. They found that the number of EMT-specific tubular epithelial cells (TECs) was closely related to serum creatinine concentration and the degree of renal interstitial damage, indicating that EMT participation Human kidney fibrosis process. Now data show that in the event of kidney disease, most of the fibroblasts in the renal interstitium are transformed by the tubular epithelial cells derived from EMT. Selectively blocking EMT can significantly improve interstitial fibrosis in obstructive nephropathy in a murine model of the gene. Thus, EMT plays an important role in renal fibrosis.

\section{The Transdifferentiation of Tubular Epithelial Cells to Mesenchymal Cells}

The main regulatory steps of EMT Recently, EMT was found to be a very complicated process regulated mainly by the following four aspects at the cellular level: ${ }^{1}$ Loss of epithelial cell adhesion function; ${ }^{\circ} \mathrm{A}$-smooth muscle actin, A-SMA) and actin rearrangement; destruction of the tubule basement membrane; $1 / 4$ enhances cell migration and infiltration.

Under normal circumstances, tubular epithelial cells through a variety of cell adhesion mechanisms closely linked to form a complete epithelial cell population. E-cadherin is a specific adhesion molecule receptor that plays a key role in maintaining the integrity and polarity of renal tubular epithelial cells. The first step in EMT is Snail, a zinc finger transcription factor that directly binds to the E-box at the start of E-cadherin, claudin and oc-culudin, thereby inhibiting their expression. B-catenin or Smad3 together activate lymphocyte augmenter and mediate Snail in lymphocyte nuclei. Presumably, the Snail transcription factor plays a key role in the early stages of EMT by inhibiting the gene expression of renal tubular epithelial cell adhesion molecules. The most commonly characterized tubular epithelial cells are marked by keratin. Interstitial cells mainly express vimentin, which is a mesenchymal marker of cells. A-SMA is a marker protein of smooth 
muscle and myofibroblasts. Actin reorganization and A-SMA expression not only signify the morphological transformation of epithelial cells, but also make them migrate, infiltrate and contract. Tubular epithelial cells and myofibrob-last are separated by the tubular basement membrane (TBM), and the rupture of TBM provides the basic conditions for the transformed cells to migrate to the interstitial space. On the other hand, intact TBM also prevents tubular epithelial cells from contacting stromal matrix components such as type I collagen, whereas epithelial cells contact with type I collagen induces EMT. Thus, TBM injury is also an indispensable link to start EMT. The main component of TBM is type IV collagen, which is a specific substrate for metallo-proteinase-2 (MMP-2) and MMP-9. Transforming growth factor-B (TGF-B) stimulates tubular epithelial cells to synthesize MMP-2 and MMP-14, of which MMP-14 is an activator of MMP-2. In the remnant kidney model, both enzymes are also detectable at the site of TBM fragmentation. In addition, tissue-type plasminogen activator (t-PA) can induce gene expression and protein secretion of MMP-9 in renal interstitial fibroblasts, thereby disrupting TBM and promoting EMT. MMP-9 was significantly decreased in t-PA-negative obstructive nephropathy rats, and the structure and function of TBM were effectively protected. Thus, both MMP-2 and MMP-14 conjugates and MMP-9 can trigger EMT by disrupting TBM.

Transformed epithelial cells inevitably have to play a role in the interstitial, so they must be infiltrating ability. We have observed that transformed cells have a strong ability to move through the damaged TBM into the interstitium. In fact, the transformed cells can simultaneously destroy TBM during the migration process. Some experiments confirm that this destruction is caused by the matrix remodeling of TBM. In addition, myofibroblasts express A-SMA and have potential systolic function. The above two aspects of interaction accelerated the transitional cells to the stromal migration. Through a series of in vitro cell culture and animal experiments, initially established the process of EMT model. Although this is only a preliminary understanding of the complicated process of EMT, it has become clear to us that the occurrence of EMT depends on the interaction between exogenous factors and various intracellular regulators.

\section{EMT's Main Regulatory Factors and the Interaction between Cytokines}

EMT by many growth factors, cytokines, hormones and extracellular matrix regulation, the specific signal transduction pathway is still not precise, which is currently one of the hot spots. TGF-B1 as a core factor, start and regulate the whole process of EMT. It is worth emphasizing that the process of TGF-B1 inducing EMT is the main path of TGF- $\beta$ leading to renal interstitial fibrosis under pathological conditions. TGF-B induces phosphorylation of Smad2 in tubular epithelial cells, which in turn leads to overexpression of Smad7, which inhibits TGF- $\beta$-mediated Smad2 activation, and inhibits EMT and collagen synthesis [8]. So some scholars have proposed Smad signaling pathway can both promote and inhibit the EMT process. It has been demonstrated that the combination of epidermal growth factor (EGF) and TGF-B promotes the transformation of tubule epithelial cells into mesenchymal cells. Like EGF, fibroblast growth factor-2 (FGF-2) decreases the expression of E-cadherin in TECs and induces the production of vimentin, FSP1 and A-SMA, thereby promoting EMT and this effect was significantly enhanced after the addition of TGF-B1. All of these factors may be involved in EMT by regulating the expression of TGF-B1. Experimental results show that TGF-B1 and bFGF-2 have positive feedback regulation in cultured tubular epithelial cell lines in vitro. Interleukin-1 (IL-1) also induces the phenotypic transformation of TEC, and this effect disappears by the addition of anti-TGF-B1 neutralizing antibody, indicating that the involvement of IL-1 in EMT is also induced by TGF-B1. It has recently been reported that MMP-2 is necessary for the induction of EMT [5] and its effect is also considered to be related to the activity of TGF-Bl. Thus, TGF-B1 is a central factor in the induction of EMT by various factors.

TGF-B can also induce the expression of TEC integrin-linked kinase (ILK) in a time-and dose-dependent manner and is regulated by the intracellular Smad signaling pathway. ILK is an intercellular serine / threonine protease that interacts with B-integrin and many other cytoskeletal proteins in the cytoplasm and is involved in the regulation of integrin-mediated processes such as cell adhesion, Changes in cell morphology, deposition of extracellular matrix (ECM). Human 
proximal tubular cells (ILs) secrete ILK to inhibit E-cadherin expression and to synthesize fibronectin (FN) and MMP-2, thereby promoting cell migration and ECM deposition. In contrast, TGF-B-induced EMT was mostly absent in ILK-negative patients. The results of animal experiments suggest that administration of hepatocyte growth factor (HGF) can block the expression of ILK, inhibit the occurrence of EMT in TEC, and improve renal interstitial fibrosis. This shows that ILK also plays an important role in EMT.

\section{The Function of the Main Effect Cells in Renal Interstitial Fibrosis}

\subsection{Mesenchymal Fibroblasts}

Because fibroblasts proliferate rapidly, producing large amounts of FN and collagen, especially type I and type III collagen, has long been thought to play a key role in renal interstitial fibrosis. In fact, however, it remains unclear until now the exact relationship between the number of fibroblasts and the severity of interstitial fibrosis, since no specific markers have been found for fibroblasts in tissue. For the first time, Strutz et al. Cloned FSP1 and in the experimental fibrosis model, the number of cells expressing FSP1 was rapidly increased. Later, some people use the herpes simplex virus thymidase to control the expression of FSP1 and selectively remove the tissue fibroblasts to improve the fibrosis. This test for the first time directly proves that fibroblasts play an important role in the fibrosis of the body.

Interstitial fibroblasts come from a wide range of sources. Kidney fibroblasts include: mesenchymal fibroblasts, circulating fibroblasts, fibroblasts transformed from epithelial cells, bone marrow-derived fibroblasts, and myofibroblasts expressing A-SMA. Interstitial fibroblasts can synthesize ECM leading to renal interstitial fibrosis; hyperplasia can also occur cell phenotype changes to become myofibroblasts. Myofibroblasts produce large amounts of type I collagen and type III collagen, expressing A-SMA, which is a terminally differentiated cell between fibroblasts and smooth muscle cells and is a major source of interstitial extracellular matrix. Myofibroblasts have been considered as activated fibroblasts. However, some scholars recently found that activated interstitial fibroblast cell adhesion molecule-1 expression was positive, but usually does not express A-SMA, so the original concept of doubt. Interstitial fibroblasts can be activated by certain cytokines such as TGF- $\beta$, PDGF and FGF-2. Some studies have shown that patients with chronic kidney disease FGF-2 renal biopsy was significantly increased, and mainly exist in interstitial fibroblasts, and through autocrine stimulation of fibroblasts for injury repair. In vitro experiments show that FGF-2 is a strong mitogen of renal fibroblasts, but it does not stimulate type I collagen or FN synthesis. In addition, fibroblasts secrete various cytokines themselves, and these cytokines act on interstitial fibroblasts, resulting in the abnormal expression of certain genes that control their growth and apoptosis, leading to overgrowth and abnormal differentiation of fibroblasts Reduce death, increase the number of cells and collagen synthesis, eventually leading to renal interstitial fibrosis.

\subsection{Macrophages}

Macrophages can secrete a variety of factors involved in renal fibrosis, and in the stroma, the number of macrophage infiltrates correlates with the severity of fibrosis. Therefore, it has been thought that macrophages can promote renal fibrosis. This argument is supported by a number of subsequent studies. However, Nishida et al. unexpectedly found that exudative macrophages have anti-fibrotic effects in renal fibrosis. After treatment of deadly bone marrow cells in irradiated wild type mice, they transplanted the kidneys of the mice with the absence of the AngII1 receptor (AT1R) gene into wild mice and unilateral ureteral ligation with untreated wild animals. Fourteen days later, the difference in renal lesions between the two groups of mice was observed. The results showed that the number of infiltrating macrophages in the former decreased but the degree of renal fibrosis was heavier. Analysis found that the treatment group due to the absence of AT1R gene lead to monocytes and macrophages in peripheral blood infiltration and phagocytic function weakened, it cannot clear excess ECM components and apoptotic cells, thereby increasing renal interstitial 
fibrosis. It has been demonstrated that urokinase cellular recep-tor (UPAR) participates in cell adhesion, invasion and angiogenesis. In the UP-AR - / - rats, the degree of renal fibrosis was more severe than that in the control group, and the number of A-SMA-expressing cells and CD34 + endothelial cells was significantly increased, while the number of interstitial macrophages was significantly decreased. This study again demonstrates that macrophages do not exacerbate renal interstitial fibrosis and may even reduce fibrosis.

\subsection{Mast Cells}

The extent of renal interstitial fibrosis is also closely related to tryptase-positive mast cells in the stroma. Experiments show that mast cell tryptase can promote renal interstitial fibroblast proliferation, synthesis of FN and type I collagen, produce ECM, and in a dose-dependent manner. Mast cells promote the proliferation of fibroblasts depends on the activity of cyclooxygenase-2 (COX -2) and prostaglandin synthesis. 5/6 Nephrectomized Animal Model Experiments have shown that mast cells promote the expression of multiple factors, including stem cell factor, IL-8 and TGF- $\beta$, to promote renal interstitial fibrosis. However, some scholars recently found that mast cells only appear in interstitial fibers with a small amount of infiltrating T-lymphocytes and macrophages, but are rare in the interstitial lesions where monocytes infiltrate. So presumably, mast cells may have potential anti-fibrosis.

\section{Conclusions}

In conclusion, we recognize that in the early stages of renal interstitial fibrosis, fibroblasts and tubular epithelial cells are activated by the RAS system; subsequent destruction of the TBM causes phenotypic transformation of epithelial cells producing large numbers of fibroblasts, Accelerate the process of fibrosis, eventually leading to end-stage renal insufficiency. EMT is one of the main mechanisms leading to renal interstitial fibrosis, some of which have been clear signal transduction pathway. Also found some can effectively fight interstitial fibrosis factor, the most prominent is BMP7 and HGF. ACE-I has recently proposed the role of anti-fibrosis. All of these provide a new idea for further research on effective measures to prevent and cure renal interstitial fibrosis.

\section{References}

[1] Li Xiaodong, Wang Baoxing. Anti-renal interstitial fibrosis molecular research [J]. Foreign Medical Sciences. Urinary System Volume 2005 (06). 62-67.

[2] Peng Yan. Hepatocyte growth factor and renal interstitial fibrosis prevention and treatment of new progress [J]. Foreign Medical Sciences. Urinary System Volume. 2004 (01) .50-78.

[3] Prevention and treatment of renal interstitial fibrosis [J]. Foreign Medical Sciences. Urinary System Volume 2005 (06).62-67.

[4] Xu Jianhua, Xiao Hongbo, Cheng Yuhua, Wang Yu, Zhang Guiling, Lu Jinlei, Shao Yi. Prevention and treatment of renal interstitial fibrosis with pirfenidone [J]. Zhongguo Yi Xue Yi Za Zhi. 2017 (19).162-167.

[5] Xiong Ying, Wang Tao. Progress in the treatment of renal interstitial fibrosis [J]. Chinese Medical Equipment. 2014 (S2) .50-67.

[6] Wang Zijie, Tan Ruoyun, Gu Min. Renal interstitial fibrosis after kidney transplantation diagnosis and treatment [J]. Renal Disease and Dialysis Kidney Transplantation. 2014 (03).12-15.

[7] Liu Yuyuan, Liu Hong. Progress in research of stem cells in renal interstitial fibrosis [J]. Chinese Journal of Integrated Traditional and Western Nephrology. 2013 (05) .50-78. 